Pacific Journal of Mathematics

DEHN'S CONSTRUCTION AND THE POINCARE IIS Foreman WeAN 


\title{
DEHN'S CONSTRUCTION AND THE POINCARÉ CONJECTURE
}

\author{
NORRIS WEAVER
}

\begin{abstract}
M. Dehn used a type of homology preserving surgery on $S^{3}$ to produce an infinite family of irreducible homology 3spheres. We apply Dehn's construction to arbitrary 3-manifolds, give groups invariant under Dehn's construction, give a reduction of the Poincaré conjecture, give a nontrivial example of links in $S^{3}$ with homeomorphic exteriors, and give a result connerning knots with property $P$.
\end{abstract}

\section{Introduction and statement of main results.}

Dehn's construction can be iterated, which gives rise to the notion of Dehn equivalence between 3-manifolds [see §2]. Dehn equivalent manifolds not only have the same homology, but we also have the following invariant groups.

TheOREM A. If $M$ and $N$ are orientable and Dehn equivalent, then $\pi_{1} M /\left(\pi_{1} M\right)_{3}$ and $\pi_{1} N /\left(\pi_{1} / N\right)_{3}$ are isomorphic, where $\left(\pi_{1} M\right)_{3}$ and $\left(\pi_{1} N\right)_{3}$ are the third lower central subgroups.

It follows from Theorem A that manifolds with the same homology need not be Dehn equivalent, e.g., $S^{1} \times S^{1} \times S^{1}$ and the connected sum $\left(S^{1} \times S^{2}\right) \#\left(S^{1} \times S^{2}\right) \#\left(S^{1} \times S^{2}\right)$.

The notion of Dehn equivalence can be stabilized as follows. Let $M^{\prime}, N^{\prime}$ be 3-manifolds. Attach finitely many 1-handles [thickend 1cells] to $\partial M^{\prime}$ and $\partial N^{\prime}$ to obtain 3-manifolds $M$ and $N$. We say say that $M^{\prime}$ and $N^{\prime}$ are stably Dehn equivalent if there exist such manifolds $M$ and $N$ which are Dehn equivalent. This is an equivalence relation. Recall that a homotopy 3-disc is a compact, simply connected 3-manifold whose boundary is homeomorphic with $S^{2}$. Our main result is the following.

THEOREM B. Every homotopy 3-disc is stably Dehn equivalent with $D^{3}$.

It follows from Theorem B that every homotopy 3-sphere [i.e., simply connected, closed 3-manifold] is a connected sum factor of a manifold which is Dehn equivalent with $S^{3}$.

Theorem B is a reduction of the Poincaré conjecture, i.e., the Poincaré conjecture can be proved by showing that $D^{3}$ is the only homotopy 3 -disc which is stably Dehn equivalent with $D^{3}$. 
One can construct 3-manifolds as follows, (i) successively perform Dehn constructions on a solid torus $T_{g}$ of genus $g \geqq 0$ a finite number, $k \geqq 1$, of times to produce a manifold $T_{g}^{\prime}$, and (ii) remove 1handles, $g$ in number, from $T_{g}^{\prime}$ to produce a manifold $M$. According to Theorem $\mathrm{B}$, if the Poincaré conjecture is false, then a counterexample $M$, i.e., a homotopy 3-disc different from $D^{3}$, can be produced by this construction, which we call the $S D$-construction.

Attempting to construct a nontrivial homotopy 3-disc by the $S D$-construction in the case $g=0, k=1$ [or in the case $g=1, k=1$ ] is the same as trying to produce a nontrivial homotopy 3 -sphere by doing a single Dehn construction on $S^{3}$. The conjecture in knot theory that all knots have property $P$ is the conjecture that a single nontrivial Dehn construction on $S^{3}$ never produces a simply connected manifold. [See $\S 2$ for the concept of a trivial Dehn construction.] This conjecture has been studied by several authors, see (1), (2), (4), (6), (7) for example, and implies that two knots in $S^{3}$ with homeomorphic exteriors have the same type. [An exterior is the complement of a open tubular or regular neighborhood.]

We consider the case $g=0, k=2$ of the $S D$-construction. Let $L=\left(l_{1}, l_{2}\right)$ be a link in $S^{3}$ with two components. Successively perform Dehn constructions along $l_{1}$ and $l_{2}$ to obtain a manifold $M$. If either Dehn construction is trivial, then up to diffeomorphism, $M$ can be produced by a single Dehn construction on $S^{3}$, so assume both Dehn constructions to be nontrivial. If $l_{1}$ and $l_{2}$ have linking number zero and $l_{1} \cup l_{2}$ is the boundary of an embedded copy of $S^{1} \times I$ in $S^{3}$, then again $M$ can be produced by a single Dehn construction, so we eliminate this case as well and call the remaining case the nontrivial case. The obvious conjecture is that $M$ is never simply connected in the nontrivial case. A counterexample to this conjecture is given as Example 3 of $\S 4$.

The counterexample above has the following significance in the theory of links. It is well known that if a link in $S^{3}$ has an unknotted component, then the exterior of the link may be homeomorphic with the exterior of a link of different type [see Example 1 of $\S 4$ ]. Let $T_{1}, T_{2}$ be closed tubular neighborhoods of two components, say $l_{1}$ and $l_{2}$, of a link with no unknotted components. If there exists an embedding

$$
\left(S^{1} \times I, S^{1} \times 0, S^{1} \times 1\right) \longrightarrow\left(S^{3} \backslash \operatorname{int}\left(T_{1} \cup T_{2}\right), \partial T_{1}, \partial T_{2}\right),
$$

then again the exterior of $L$ may be homeomorphic with the exterior of a link of different type [see Example 2 of $\S 4$ ]. However, in this case one of the nontrivial knots $l_{1}, l_{2}$ must be a cable about the other, and consequently one of $l_{1}, l_{2}$ is a companion of the other. Example 3 of $\S 4$ proves the following. 
TheOREM C. There exists a [tame] link in $S^{3}$ such that (i) the exterior is homeomorphic with the exterior of another link of different type, and (ii) no component is a companion of another component.

The next four sections deal respectively with the definition of Dehn equivalence and the proofs of Theorems A, C, and B, together with related results. It is possible to obtain the same homology sphere from $S^{3}$ by Dehn constructions along different knots. In the final section we give an infinite class of such knots and verify that most of the homology spheres involved are not simply connected, which generalizes a result of (2) and (4).

In the following we work in the category of smooth manifolds and maps since this is convenient for the transversality argument in $\S 5$. Everything we do has a $P L$ analog however.

2. Dehn's construction. Let $M$ be a 3-manifold and let $*$ be an element of $S^{1}$. Let $f: S^{1} \times D^{2} \rightarrow$ int $M=M \backslash \partial M$ be an embedding such that $f\left(S^{1} \times *\right)$ is null-homologous in $M \backslash$ int $f\left(S^{1} \times D^{2}\right)$. Let $h: S^{1} \times \partial D^{2} \rightarrow f\left(S^{1} \times \partial D^{2}\right)$ be a diffeomorphism such that $h\left(S^{1} \times *\right)=$ $f\left(S^{1} \times *\right)$. Glue $S^{1} \times D^{2}$ to $M \backslash$ int $f\left(S^{1} \times D^{2}\right)$ by $h$. The resulting manifold $M_{1}$ has been obtained from $M$ by Dehn's construction along $f\left(S^{1} \times 0\right)$. The construction is trivial if either $f\left(S^{1} \times 0\right)$ bounds an embedded disc in $M$ or $h\left(* \times \partial D^{2}\right)$ is null-homotopic in $f\left(S^{1} \times D^{2}\right)$. A trivial Dehn construction does not change the diffeomorphism class of $M$. If $M_{0}, M_{1}, \cdots, M_{n}, n \geqq 0$ are such that $M_{i+1}$ is obtained from $M_{i}$ by Dehn's construction, then we say that any two manifolds diffeomorphic with $M_{0}$ and $M_{n}$ respectively are Dehn equivalent. This is an equivalence relation. It is easily proved that there is a commutative square as follows.

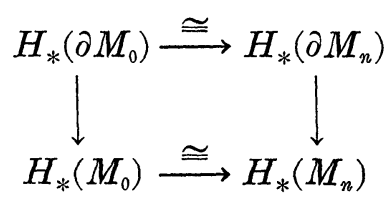

3. Proof of Theorem A and related results. Let $M, M_{1}, f, h$ be as in $\S 2$ and assume $M$ to be orientable. Let $F$ be a compact surface in $M \backslash$ int $f\left(S^{1} \times D^{2}\right)$ with $\partial F=f\left(S^{1} \times *\right)$. Let $P$ be a closed $\varepsilon$-neighborhood of $F \cup f\left(S^{1} \times D^{2}\right)$ in $M$. Then $P \cong F \times I$ after smoothing, and $\pi_{1} \partial P$ has generators $a_{i}, b_{i}, a_{i}^{\prime}, b_{i}^{\prime}, 1 \leqq i \leqq g$ and the defining relation

$$
\left[a_{1}, b_{1}\right] \cdots\left[a_{g}, b_{g}\right]=\left[a_{1}^{\prime}, b_{1}^{\prime}\right] \cdots\left[a_{g}^{\prime}, b_{g}^{\prime}\right]
$$


where $g$ is the genus of $F$. [We use the definition $[x, y]=x y x^{-1} y^{-1}$.] The Dehn construction producing $M_{1}$ from $M$ is simultaneously a Dehn construction producing a manifold $P_{1}$ from $P$. Presentations of $\pi_{1} P$ and $\pi_{1} P_{1}$ can be obtained from the presentation of $\pi_{1} \partial P$ by adding the relations (i) $a_{i}=a_{i}^{\prime}, b_{i}=b_{i}^{\prime}$, and (ii) $d^{n} a_{i} d^{-n}=a_{i}^{\prime}, d^{n} b_{i} d^{-n}=b_{i}^{\prime}$ respectively, where $d$ denotes $\left[a_{1}, b_{1}\right] \cdots\left[a_{g}, b_{g}\right]$ and the integer $n$ is, up to sign, the intersection number of $f\left(* \times S^{1}\right)$ and $h\left(* \times S^{1}\right)$ in $f\left(S^{1} \times \partial D^{2}\right)$. Given any group $G$ we denote $G / G_{c+1}, c \geqq 0$ by $V_{c}(G)$. We have a commutative diagram

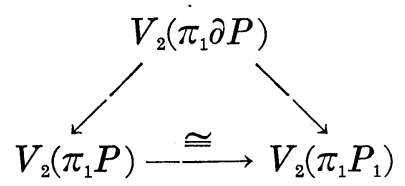

in which the slanted arrows are inclusion induced. Note that $V_{c}$ is a functor. The image of this functor is the group variety $V_{c}$ of groups with nil-c. The image of a push out diagram under the functor $V_{c}$ is a push out diagram in the category $V_{c}$. The SeifertVanKampen theorem applied to $M=P \cup(M \backslash$ int $P), M_{1}=P_{1} \cup(M \backslash$ int $P)$ describes $\pi_{1} M$ and $\pi_{1} M_{1}$ as push outs. By the commutative triangle above these push out diagrams become isomorphic after the functor $V_{2}$ is applied. Thus $V_{2}\left(\pi_{1} M\right) \cong V_{2}\left(\pi_{1} M_{1}\right)$ and Theorem $\mathrm{A}$ is proved.

It follows from this proof that the diagram of $\S 2$ also commutes when $H_{*}$ is replaced by $V_{2} \pi_{1}$.

We show that $V_{3}\left(\pi_{1} M\right)$ is not necessarily invariant under Dehn equivalence by giving an example. Let $P, P_{1}$ be as in the proof above, but choose $P, P_{1}$ so that $g, n=1$. Let $N$ be the double of $P$, and glue $\partial P$ to $\partial P_{1}$ by the identity map to obtain $N_{1}=P \cup P_{1}$. Then $N$ and $N_{1}$ are Dehn equivalent, but $V_{3}\left(\pi_{1} N\right)$ is nilpotent of class three while $V_{3}\left(\pi_{1} N_{1}\right)$ is nilpotent of class two.

If $F$, as in the proof of Theorem A, is chosen to have the smallest possible genus $g$, then we say that the Dehn construction has genus $g$. Let $E$ be the group variety defined by the Engel law $[x, x, y]=1$. [We define $[x, y, z]$ to be $[x,[y, z]]$. $]$ The proof of Theorem A with slight modification proves the following.

THEOREM 1. If $N$ is obtained from $M$ by a Dehn construction of genus one, then $E\left(\pi_{1} M\right) \cong E\left(\pi_{1} N\right)$.

The groups in $E$ have the following structure.

THEOREM 2. Let $S$ be a set which generates a group G. Then $E(G)$ is isomorphic with $V_{3}(G)$ modulo the relations $[a, b, c][b, a, c]=1$, $a, b, c \in S$. Also $E(G)$ is an extension of an abelian group of period 
3, namely $E(G)_{3}$, by $V_{2}(G)$.

The proof of Theorem 2 has much in common with the proof of the structure theorem for Burnside groups of period three (5, pp. 320-324), so we omit the proof.

It follows from Theorem 2 that if $G$ has a given finite presentation, then $E(G)$ has a solvable word problem.

The group $E\left(\pi_{1} M\right)$ is not necessarily invariant under Dehn equivalence. This is shown by manifolds $N, N_{1}$ constructed as above, but in the case $g \geqq 2, n=1$ rather than the case $g, n=1$.

4. Proof of Theorem C. Let $M, M_{1}, f, h$ be as in $\S 2$. Orient $S^{1} \times \partial D^{2}$ and $f\left(S^{1} \times \partial D^{2}\right)$ as the boundaries of $S^{1} \times D^{2}$ and $f\left(S^{1} \times D^{2}\right)$ respectively. Assume that $M$ is oriented and that $f, h$ are orientation preserving. Then $M_{1}$ has a canonical orientation, and we say that the Dehn construction has type $(l, n)$ where $l$ is the unoriented curve $f\left(S^{1} \times 0\right)$ and $n$ is the intersection number of $f\left(* \times \partial D^{2}\right)$ with $h\left(* \times \partial D^{2}\right)$ in $f\left(S^{1} \times \partial D^{2}\right)$. [Thus if $n=1$, the oriented curves $h\left(* \times \partial D^{2}\right)$ and $f\left(S^{1} \times 0\right)$ are isotopic in $f\left(S^{1} \times D^{2}\right)$.] Up to orientation preserving diffeomorphism $M_{1}$ is determined by $n$ and the isotopy class of $l$ in $M$.

Let $K$ be the link of Figure 1. Let $k_{1}$ be the small unknotted component, let $k_{2}$ be the component which is a figure eight knot, and let $k_{3}$ be the remaining unknotted component. The example which proves Theorem $\mathrm{C}$ will be constructed in three steps each of which is an example.

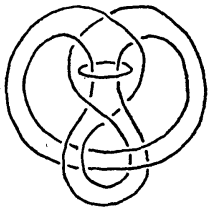

Figure 1

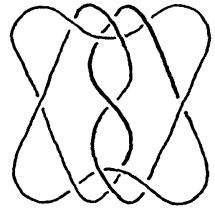

FIGURE 2

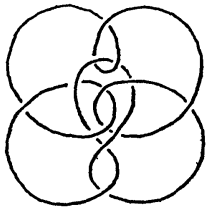

FiguRe 3

EXAMPLE 1. A Dehn construction of type $\left(k_{1}, 1\right)$ on $S^{3}$ has genus zero and changes $K$ into a link $K^{\prime}$ whose components $k_{2}^{\prime}$ and $k_{3}^{\prime}$ are parallel figure eight knots. Thus $K$ and $K^{\prime}$ have different types and diffeomorphic exteriors. Note that the Dehn construction $\left(k_{1}, 1\right)$ has genus 1 when considered tohbe a Dehn construction on the exterior of $k_{3}$, and it changes this exterior into the exterior of $k_{3}^{\prime}$.

ExAmple 2. Let $n \neq 0$ be an integer. Do Dehn constructions of types $\left(k_{2}^{\prime}, n\right)$ and $\left(k_{3}^{\prime},-n\right)$ simultaneously to change $K^{\prime}$ into a link $K^{\prime \prime}$. [These two constructions can also be performed successively, 
but it is conceptually and notationally convenient to perform such constructions simultaneously.] The components $k_{2}^{\prime \prime}$ and $k_{3}^{\prime \prime}$ are figure eight knots while $k_{1}^{\prime \prime}$ has Alexander polynomial $n t^{2}+(1-2 n) t+n$. Thus $K, K^{\prime}, K^{\prime \prime}$ are of different types, but their exteriors are diffeomorphic. [Note that if we tie a small knot in $k_{1}^{\prime}$ before doing the construction of $K^{\prime \prime}$, then we obtain links with different types, no unknotted components, and diffeomorphic exteriors.]

EXAMPLE 3. A Dehn construction of type $\left(k_{3},-n\right)$ changes $K$ into a link with components $l_{1}, l_{2}, l_{3}$. Delete $l_{3}$ to obtain $L$. The components of $L$ have polynomials $n t^{2}+(1-2 n) t+n$ and $t^{2}-3 t+1$ respectively. If $n \neq-1$, then neither polynomial divides the other, so neither component of $L$ is a companion knot of the other component. Delete $k_{3}^{\prime \prime}$ from $K^{\prime \prime}$ to obtain $L^{\prime}$ whose components have polynomials $n t^{2}+(1-2 n) t+n$ and $t^{2}-3 t+1$ respectively. Neither component of $L^{\prime}$ is a companion of the other if $n \neq-1$. By the construction of $L$ and $L^{\prime}$ we can transform $L$ into $L^{\prime}$ by successively doing Dehn constructions of types $\left(l_{3}, n\right)$ and $\left(k_{1}, 1\right)$ followed by simultaneously doing the constructions $\left(k_{2}^{\prime}, n\right)$ and $\left(k_{3}^{\prime},-n\right)$. The Dehn constructions $\left(l_{3}, n\right)$ and $\left(k_{3}^{\prime},-n\right)$ cancell each other out, so the simultaneous constructions $\left(l_{1}, 1\right)$ and $\left(l_{2}, n\right)$ transform $L$ into $L^{\prime}$. Consequently $L$ and $L^{\prime}$ have diffeomorphic exteriors. It remains to show that $L$ and $L^{\prime}$ are of different types. This is not easy [even though $l_{1}, l_{2}, l_{1}^{\prime}, l_{2}^{\prime}$ all seem to have different types], so we give a proof only in the case $n=1$. In the case $n=1$ the components of $L$ are a trefoil knot and the knot of Figure 2 while $l_{1}^{\prime}, l_{2}^{\prime}$ are respectively the knot of Figure 3 and a figure eight knot. We can distinguish $l_{1}$ from $l_{1}^{\prime}$ as follows. Let $G_{i}, i=0,1$ be the knot groups of $l_{1}, l_{1}^{\prime}$ respectively. Let $m_{i} \in G_{i}$ be a meridian element. Let $H_{i}$ be $G_{i}$ modulo the relation $m_{i}^{2}=1$. Note that $H_{i}$ does not depend on the choice of $m_{i}$. Let $N_{i}$ be the kernel of the canonical epimorphism $H_{i} \rightarrow Z_{2}$. Let $A_{i}$ be the abelianization of the commutator subgroup of $N_{i}$. Present $A_{i}$ by the Reidemeister-Schreier algorithm to show that $A_{0}$ is trivial while $A_{1} \cong Z_{8} \oplus Z_{8}$. Thus $l_{1}$, $l_{1}^{\prime}$ have different types and Theorem $\mathrm{C}$ is proved.

5. Proof of Theorem B. We prove the following Theorem 3 which clearly implies Theorem B.

THEOREM 3. Let $M$ be a compact, connected, and orientable 3manifold. If $\partial M$ is connected and $\pi_{1}(M, \partial M)$ is trivial, then $M$ is stably Dehn equivalent with $D^{3}$. The stable equivalence can be taken to only use Dehn constructions of genus one. 
Theorem 2 is proved by using link homotopy in a relative case. A relative link is an embedding $(U, \partial U) \rightarrow(M, \partial M)$ where $U$ is a finite disjoint union of closed intervals. A link homotopy is a homotopy $H:(U, \partial U) \times I \rightarrow(M, \partial M)$ between relative links such that distinct components of $U$ do not intersect each other during the homotopy, but self-intersections of components are allowed. By transversality theory we can approximate $H$ by a link homotopy $H^{\prime}$ such that (i) $h_{0}^{\prime}=h_{0}, h_{1}^{\prime}=h_{1}$, (ii) each $h_{t}^{\prime}$ is an immersion [meeting $\partial M$ transversely] with at most one self-intersection, and (iii) the induced map

$$
(U \times U \backslash \delta U) \times I \longrightarrow M \times M
$$

is transverse to the diagonal $\delta M$. In the following we will only use link homotopies with the properties (ii) and (iii), and we assume $M$ to be as in Theorem 3. Let $H$ be a link homotopy. We claim that the exteriors of $h_{0}$ and $h_{1}$ are Dehn equivalent. Note that $h_{t}$ has a self-intersection for only finitely many values of $t$. The exterior of the link just before a self-intersection can be changed into the exterior just after the self-intersection by a Dehn construction of genus one which is simultaneously a Dehn construction of genus zero on $M$. [The situation is essentially that of the Dehn construction of Example 1 of $\S 4$ which changes the exterior of $k_{3}$ into the exterior of $k_{3}^{\prime}$.] The claim is proved. Now we claim that any relative link in $M$ is link homotopic with an isotopy trivial relative link. [A relative link is isotopy trivial if (i) the components lie in disjoint open sets each of which is diffeomorphic with $\boldsymbol{R}^{2} \times[0, \infty)$, and (ii) in each copy of $R^{2} \times[0, \infty)$ the link component is given by $x=0$, $y^{2}+z^{2}=1, z \geqq 0$.] In order to prove the claim by induction we temporarily drop the assumption that $M$ be compact. Clearly the claim is true if $U$ is connected. Assume the claim to be true when $U$ has $n \geqq 1$ components and consider the case $n+1$. We can link homotop one component so that it becomes isotopy trivial. Extend this link homotopy to become a link homotopy of the entire link. Remove the isotopy trivial component from $M$ to produce a manifold, say $M \backslash l_{1}$. By inductive hypothesis we can link homotop the remaining components in $M \backslash l_{1}$ to become an isotopy trivial link. The entire link is now isotopy trivial in $M$ and the claim follows. The two claims prove Theorem 2 as follows. The compact manifold $M$ has some handlebody decomposition

$$
M \cong T_{g} \cup h_{1} \cup \cdots \cup h_{t}, \quad 0 \leqq t \leqq g, \quad g \geqq 0
$$

where $T_{g}$ us a solid torus of genus $g$ and the $h_{i}$ 's are disjoint 2handles, i.e., disjoint thickened 2-cells attached to $\partial T_{g}$. [Sometimes 
2-handles on a 3-manifold are called pillboxes.] The union $h_{1} \cup \cdots \cup h_{t}$ is a closed tubular neighborhood of a relative link. This relative link can be link homotoped to become isotopy trivial. Hence $M \backslash$ int $T$, where $T$ is a closed tubular neighborhood of an isotopy trivial link with $t$ components, is Dehn equivalent with $T_{g}$. Since $M$ can be obtained from $M \backslash$ int $T$ by removing 1-handles, the theorem is proved.

6. The manifolds $B(p, q, r)$. Many examples of diffeomorphic manifolds obtained by Dehn constructions along different knots can be given as follows. Select an order $l_{1}, l_{2}, l_{3}$ for the components of a Borromean link. Let $B(p, q, r), p, q, r$ any integers, be the manifold obtained from $S^{3}$ by simultaneously doing Dehn constructions of type $\left(l_{1}, p\right),\left(l_{2}, q\right)$, and $\left(l_{3}, r\right)$. Diffeomorphisms of $S^{3}$ which map the Borromean link onto itself induce orientation preserving diffeomorphisms

$$
\begin{aligned}
& B(\pi(p), \pi(q), \pi(r)) \cong B(p, q, r) \\
& B(-p,-q,-r) \cong-B(p, q, r)
\end{aligned}
$$

where $\pi$ is a permutation of $p, q, r$ and $-B(p, q, r)$ is $B(p, q, r)$ with reversed orientation. We can obtain $B(p, q, r)$ by first doing the $\left(l_{1}, p\right)$ and $\left(l_{2}, q\right)$ constructions to change $l_{3}$ into a knot $k(p, q)$ in $S^{3}$ and then doing the Dehn construction of type $(k(p, q), r)$. By $(*)$ the Dehn constructions $(k(p, q), r),(k(q, r), p)$ and $(k(r, p), q)$ all produce the same manifold from $S^{3}$. The knots $k(p, q), k(q, r), k(r, p)$ are different in general since $k(p, q)$ has Alexander polynomial $p q t^{2}+$ $(1-2 p q) t+p q$.

The question of whether any of the $B(p, q, r)$ 's with $p, q, r \neq 0$ are simply connected naturally arises. The following theorem is a partial answer.

THEOREM 4. The group $\pi_{1} B(p, q, r), p, q, r \neq 0$ is nontrivial if none of the absolute values $|p|,|q|,|r|$ is 2 or 3 .

Proof. The knot $k(1, q), q \neq 0$ has property $P$ according to (2) and (4). The theorem follows if any of $|p|,|q|,|r|$ is 1 . The remaining case is $|p|,|q|,|r|>3$, and we can assume $r>0$. Present $\pi_{1} B(p, q, r)$ as

$$
\left\langle a, b, c ; a\left[b^{-1}, c\right]^{p}, b\left[c^{-1}, a\right]^{q}, c\left[a^{-1}, b\right]^{r}\right\rangle .
$$

Consider the group

$$
G=\left\langle a, b, c ; a=\left[b^{-1}, c\right], c=\left[a^{-1}, b\right]\right\rangle .
$$

After the substitutions $b=a^{-1} d^{-1}$ and $a=e^{-1} d$ we obtain 


$$
G=\left\langle d, e ; d^{3}=e^{3}\right\rangle \text {. }
$$

Add the relation $d^{3}=1$ to $G$ to obtain a group in which the relations of $\pi_{1} B(p, q, r)$ become $\left(d e^{-1}\right)^{3 p+1},(d e)^{3 q+1}$, and $[d, e]^{r+1}$. Thus $\pi_{1} B(p, q, r)$ has the group

$$
\left\langle d, e ; d^{3}, e^{3},\left(d e^{-1}\right)^{3 p+1},(d e)^{3 q+1},[d, e]^{r+1}\right\rangle
$$

as a quotient. By Theorem 1 of [3] this group is nontrivial when $|p|,|q|, r>3$, so we are done.

Finally it may be noted that the $B(1,1, r)$ 's with $r \neq 0$ are the irreducible homology 3 -spheres constructed by Dehn. It might be asked if all of the $B(p, q, r)$ 's are irreducible.

\section{REFERENCES}

1. R. H. Bing, Necessary and sufficient conditions that a manifold be $S^{3}$, Ann. of Math., 68 (1958), 17-37.

2. R. H. Bing and J. M. Martin, Cubes with knotted holes, Trans. Amer. Math. Soc., 155 (1971), 217-231.

3. J. L. Britton, Solution to the word problem for certain types of groups I, Proc. Glascow Math. Soc., 3 (1956), 45-54.

4. F. González-Acuña, Dehn's construction on knots, Bol. Soc. Mat. Mex., 15 (1970), $58-79$.

5. M. Hall, The theory of Groups, Macmillan, New York, 1959.

6. R. Riley, Knots with parabolic property P, Quart. J. Math. Oxford (2) 25 (1974), 273-283.

7. Simon, On knots with nontrivial interpolating manifolds, Trans. Amer. Math. Soc., 160 (1971), 467-473.

Received June 7, 1977.

ADDIS ABABA UNIVERSITY

ADDIS ABABA, ETHIOPIA 



\section{PACIFIC JOURNAL OF MATHEMATICS}

EDITORS

RICHARD ARENS (Managing Editor)

University of California

Los Angeles, California 90024

C. W. Curtis

University of Oregon

Eugene, OR 97403

C. C. MOORE

J. DUGUNDJI

Department of Mathematics University of Southern Californa Los Angeles, California 90007

R. Finn AND J. Milgram Stanford University Stanford, California 94305

University of California

Berkeley, CA 94720

\section{ASSOCIATE EDITORS}

E. F. BeCKENBACH

B. H. NeUMANN

F. WOLF

K. YosHida

\section{SUPPORTING INSTITUTIONS}

UNIVERSITY OF BRITISH COLUMBIA UNIVERSITY OF SOUTHERN CALIFORNIA CALIFORNIA INSTITUTE OF TECHNOLOGY UNIVERSITY OF CALIFORNIA MONTANA STATE UNIVERSITY UNIVERSITY OF NEVADA, RENO STANFORD UNIVERSITY UNIVERSITY OF TOKYO UNIVERSITY OF UTAH NEW MEXICO STATE UNIVERSITY WASHINGTON STATE UNIVERSITY OREGON STATE UNIVERSITY UNIVERSITY OF WASHINGTON UNIVERSITY OF OREGON OSAKA UNIVERSITY 


\section{Pacific Journal of Mathematics \\ Vol. 73, No. 1 \\ March, 1977}

Thomas Robert Berger, Hall-Higman type theorems. $V \ldots \ldots \ldots \ldots \ldots \ldots \ldots$

Frank Peter Anthony Cass and Billy E. Rhoades, Mercerian theorems via

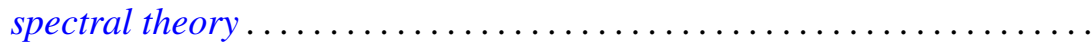

Morris Leroy Eaton and Michael David Perlman, Generating $\mathrm{O}(n)$ with

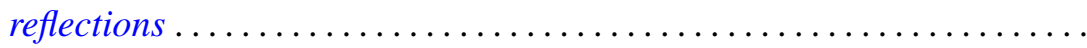

Frank John Forelli, Jr., A necessary condition on the extreme points of a

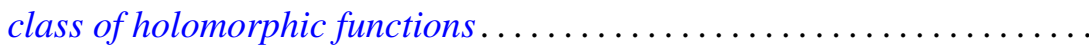

Melvin F. Janowitz, Complemented congruences on complemented

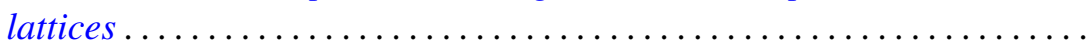

Maria M. Klawe, Semidirect product of semigroups in relation to amenability, cancellation properties, and strong $F \phi$ lner conditions....

Theodore Willis Laetsch, Normal cones, barrier cones, and the "spherical image" of convex surfaces in locally convex spaces ................

Chao-Chu Liang, Involutions fixing codimension two knots.............

Joyce Longman, On generalizations of alternative algebras .............

Giancarlo Mauceri, Square integrable representations and the Fourier

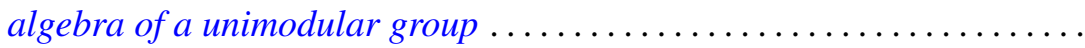

J. Marshall Osborn, Lie algebras with descending chain condition...

John Robert Quine, Jr., Tangent winding numbers and branched

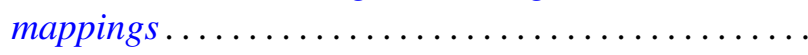

Louis Jackson Ratliff, Jr. and David Eugene Rush, Notes on ideal covers and associated primes .

H. B. Reiter and N. Stavrakas, On the compactness of the hyperspace of faces.

Walter Roth, A general Rudin-Carlson theorem in Banach-spaces ..

Mark Andrew Smith, Products of Banach spaces that are uniformly rotund in every direction.

Roger R. Smith, The R-Borel structure on a Choquet simplex ...

Gerald Stoller, The convergence-preserving rearrangements of real infinite series. ...

Graham H. Toomer, Generalized homotopy excision theorems modulo a Serre class of nilpotent groups...

Norris Freeman Weaver, Dehn's construction and the Poincaré conjecture....

Steven Howard Weintraub, Topological realization of equivariant intersection forms... 